\title{
ESTUDIOS
}

\section{El cambio en los patrones de cohesión en España en el período 2007-2009 y su repercusión entre los usuarios de servicios sociales}

\section{Domingo Carbonero Muñoz'}

Resumen: La literatura referida a los Regímenes de Bienestar apunta al frágil equilibrio entre el empleo, la familia y la protección social. A través de las Encuestas de Población Activa (EPA), las encuestas de Condiciones de Vida (ECV) y las encuestas FOESSA se profundiza en los cambios experimentados por parte de la población española, ofreciendo una comparación con los hogares y personas que alguna vez han utilizado los servicios sociales en aquellos ámbitos relativos al mercado de trabajo, la protección familiar o la cobertura desempeñada por parte de los servicios de protección social.

Este trabajo pretende indagar en el impacto que ha tenido el primer período de crisis en España, comprendido entre 2007-2009, y en qué medida ha repercutido en el aumento de la brecha entre usuarios y no usuarios.

Palabras clave: Regímenes de bienestar, pobreza, usuarios de servicios sociales, condiciones de vida

Fecha de recepción: 19 de febrero de 2015.

Fecha de admisión definitiva: 15 de abril de 2015.

\footnotetext{
' Departamento de Derecho. Área de Trabajo Social y Servicios Sociales. Universidad de La Rioja. domingo.carbonero@unirioja.es
} 


\section{The change in the bosses of cohesion in Spain in the period 2007-2009 and his repercussion between the users of social services}

Abstract: The Welfare Regimes system's literature designates the fragile balance between employment, family and social protection. Through surveys of exclusion by the Active Population Survey (EPA), living Condition Survey (ECV) and FOESSA Foundation survey, it has investigated the most important changes in households and people who have used the social services. These analyses have compared social services users and non social services users in areas like demographic, employment, family, housing, health and social protection.

The results of the EPA and the ECV serve to interpret the changes in the labour market and dwelling. As a result of these dynamics, the final conclusions note to the worsening of the living conditions in social services users in areas like demographic, employment, family, housing, health and social protection.

This study aims to investigate the impact that it has had the first period of crisis in Spain between 2007-2009 and the increase gap between users and non-users.

Keywords: welfare system, poverty, users of social services, living conditions.

\section{Le changement chez les modèles de cohésion en Espagne pendant la période 2007-2009 et sa répercus- sion entre les usagers des services sociaux}

Resumé: La littérature visant les régimes de welfare met en joue à l'équilibre fragile entre l'emploi, la famille et la protection sociale. À travers des enquêtes de population active (EPA), les enquêtes de conditions de vie (ECV) et les enquêtes dévéloppées par la Fondation FOESSA, on peut approfondir dans les changements expérimentés par une partie de la population espagnole, en offrant une comparaison avec les foyers et les personnes qui ont utilisé quelque fois les services sociaux dans les domaines relatifs au marché du travail, la protection familiale ou la couverture dégagée de la part des services de protection sociale.

Ce travail essaie d'étudier l'impact qui la première période de crise, comprise entre 2007-2009, a eu en Espagne, et dans quelle mesure il a répercuté surl'augmentation de la brèche entre des usagers et les non usageurs.

Mots-clés: régimes de welfare, pauvreté, usageurs de services sociaux, conditions de vie. 


\section{El análisis de la pobreza y de la exclusión social en España en el marco de la teoría de los regímenes de bienestar}

Una parte importante de la literatura referida a los regímenes de bienestar se ha enfocado al estudio de la pobreza y del bienestar social en Europa. En este marco de investigación, los análisis se han sustentado en las interacciones entre el mercado, la familia y el estado, comparando diferentes países europeos (Esping Andersen, G. 2002, 2007).Atendiendo al grado de desmercantilización (reducción de la dependencia del mercado de trabajo) y desfamiliarización (reducción de la dependencia respecto ayuda desempeñada por parte de la familia) se han identificado los modelos de bienestar conservador, liberal y social demócrata (Esping Andersen, 2002; Marshally Bottomore, 1950; Rodríguez y Navarro, 2006).

Los países del sur de Europa se ubican dentro del modelo conservador, presentando peculiaridades sobre el papel de la ayuda desempeñada por la familia, el mercado de trabajo y las políticas de bienestar. Este marco de estudio será aplicado al estudio de la pobreza y de la exclusión social en España, así como las tendencias experimentadas entre 2007-2009.

La familia como institución encargada de desempeñar las funciones de apoyo, protección de los más desfavorecidos y reproducción de las desigualdades ha sido un tema recurrente en los trabajos clásicos de pobreza y de bienestar (Laparra, 2002; Paugam, 2007; Esping Andersen, 2005). Históricamente, el análisis de la familia se ha caracterizado por la fuerte división del trabajo entre los ámbitos público y privado, así como el modelo sustentado en un único proveedor. En la actualidad, este patrón ha experimentado cambios importantes, explicado por la incorporación de la mujer al mercado de trabajo y el aumento del número de proveedores en el hogar, hallándose los hogares que cuentan con un único ingreso entre aquellos con mayores posibilidades de situarse debajo del umbral de la pobreza².

La transmisión intergeneracional, segundo rasgo característico de la pobreza en el sur de Europa, se ha interpretado como aspecto clave en la explicación de las trayectorias laborales, la movilidad en el sistema educativo y en el mercado de trabajo. Las referencias teóricas más importantes han recogido este hecho como expresión de la pobreza tradicional, persistente aún en nuestros días (Esping Andersen, 2002, 2005; Paugam, 2007; Castel, 1997; Del Pino y Bericat, 1998; Bourdieu y Passeron, 1969).

\footnotetext{
${ }^{2}$ Las tasas de riesgo de pobreza más elevadas se producen en aquellos hogares formados por un adulto como sustentadores del hogar con uno o más niños dependientes a su cargo. http://www.ine. es/jaxiBD/tabla.do
} 
La formación del mercado de trabajo ha sido el tercer rasgo del cual ha dado cuenta la literatura. Las características del proceso de modernización subrayan la extensión de las relaciones asalariadas como aspecto de necesaria comprensión en la transición de las sociedades tradicionales a las sociedades modernas (Durkheim, 1987). Sin embargo, el retraso de estos procesos, comparativamente al resto de países europeos, es un aspecto común de la pobreza de los países del sur de Europa reflejado en las elevadas tasas de desempleo y de precariedad en el mercado de trabajo (Castel, 1997).

De diferente naturaleza, pero dentro de este mismo apartado, se sitúan los desequilibrios entre la familia y el mercado de trabajo. En esta línea de investigación se encuentran los trabajos realizados por Taylor-Gooby (2004), quien sitúa el frágil equilibrio entre la conciliación del mundo laboral y familiar como un rasgo propio de las sociedades posmodernas.

El último rasgo a tener en cuenta en la formación de los regímenes de bienestar ha sido la protección desempeñada por el Estado social (Rodríguez, 2005). Las apreciaciones más importantes en este campo han destacado el retraso español en materia de política social y las diferencias entre las políticas universales y asistenciales. No obstante, el sistema nacional de salud (SNS) y el sistema educativo han sido piezas importantes en la formación del Estado de bienestar español, siendo el universalismo y el carácter redistributivo sus dos características fundamentales.

En cuanto al concepto de servicios sociales, su empleo tiene lugar durante el período de la transición, manejando tres criterios a este respecto: el ámbito del desempeño profesional por parte de los trabajadores sociales, al conjunto de las actividades destinadas a la provisión de bienestar social (educación, sanidad, justicia, empleo, seguridad social, vivienda y servicios sociales) o sólo esta última rama.

A pesar del intento de conformar un sistema de protección universal, denominado sistema público de servicios sociales, éste surge para sustituir los servicios de la Red de Beneficencia, de la Red de Asistencia Social y de la Red de Seguridad Social. Por lo tanto, la labor de los servicios sociales se ha ubicado en un nivel diferente en el marco de protección social, limitándose a aquellos grupos de población que presentan mayores dificultades (Casado, 1996; Rodríguez, 2005).

Siguiendo el enfoque de Sarraceno (2004), los usuarios de los servicios sociales se han estudiado mediante los riesgos o las necesidades específicas de esta población, comparadas con el conjunto de la población española. A partir de las variables de origen familiar, la inserción en el mercado de trabajo, la solidaridad familiar, los problemas de salud o la protección social, el artículo trata de ofrecer 
una visión comparada de los riesgos de exclusión social entre los usuarios de los servicios sociales, así como del riesgo experimentado por este grupo de población en este período de tiempo.

Siguiendo esta premisa, el enfoque de regímenes de bienestar es de utilidad para interpretar las necesidades de la población española y su comparación con los usuarios de los servicios sociales, puesto que encontramos una explicación global de la articulación entre familia, trabajo y protección social. Las cuestiones tratadas en este trabajo nos acercan a la protección desempeñada por los servicios sociales hacia la población que padece algún problema de exclusión social, así como la cobertura de los servicios de protección universal hacia la población que ha utilizado los servicios sociales.

\section{Principales hipótesis y objetivos}

Este trabajo ha prestado atención a las necesidades de los usuarios de los servicios sociales y a los cambios producidos entre 2007 y 2009 . Este artículo pretende mostrar el cambio de necesidades a consecuencia del primer impacto de la crisis y de las especificidades experimentadas por parte de los usuarios de los servicios sociales. Para abordar estas cuestiones, se ha formulado la siguiente hipótesis y sus objetivos correspondientes.

Hipótesis 1. "Las cambios en el ámbito de las desigualdades sociales y los problemas de exclusión social se manifiestan de manera más intensa entre los usuarios de los servicios sociales".

- Objetivo 1. Comparar los riesgos de exclusión entre los hogares y los individuos beneficiarios y no beneficiarios de los servicios sociales.

- Objetivo 2. Analizar las diferencias en la composición demográfica de los hogares e individuos beneficiarios y no beneficiarios de los servicios sociales.

La estructura del resto del trabajo se ha diferenciado en tres partes. La primera de ellas recoge las fuentes de información empleadas, las dimensiones, las sub dimensiones, los indicadores, las ventajas e inconvenientes encontrados y la técnica de análisis empleada en el estudio de las condiciones de vida de los usuarios de los servicios sociales. El segundo y el tercer apartado abordan la evolución de los usuarios de servicios sociales durante el período 2007-2009, sus riesgos y las diferencias con la población no usuaria de los servicios sociales. 


\section{Las unidades de medición empleadas en el estudio de las condiciones de vida de los usuarios de servicios sociales}

\section{I. Unidades y características de la muestra}

A partir de las series de la EPA (Encuesta de población activa) y las ECV-INE (Encuestas de condiciones de vida) se han estudiado las necesidades de la población española en el mercado de trabajo y el alojamiento. En primer lugar, la EPA es la fuente más apropiada en el estudio del mercado de trabajo. A partir de ella, se ha indagado en cuestiones relativas a la tasa de desempleo, su evolución o la repercusión de este fenómeno entre las personas con niveles educativos más bajos. En segundo lugar, las ECV-INE se utilizan en la medición de la pobreza relativa y de los problemas residenciales, haciendo referencia a indicadores tales como la tasa de pobreza, la presencia de equipamientos en el hogar o la gravedad de los problemas de salud.

Finalmente, mediante la utilización de las encuestas sobre el perfil de la exclusión social, realizadas por la fundación FOESSA se ha obtenido una muestra representativa de la población pobre y en peores condiciones de vida en España (Laparra y Pérez (Coord.), 2010). La investigación de las condiciones de vida y la labor desempeñada por parte del sistema de protección social se han realizado mediante la sub muestra de beneficiarios de los servicios sociales (Tabla 1). La realización del trabajo de campo tuvo lugar durante los años 2007 y 2009.

\section{TABLA I. Hogares e individuos que utilizan o han utilizado alguna vez los servicios sociales}

\begin{tabular}{|l|c|c|}
\cline { 2 - 3 } \multicolumn{1}{c|}{} & Encuesta 2007 & Encuesta 2009 \\
\hline Hogares encuestados & 3.418 & 3.603 \\
\hline Individuos encuestados & 9.106 & 9.590 \\
\hline $\begin{array}{l}\text { Hogares que utilizan o han utilizado alguna } \\
\text { vez los servicios sociales }\end{array}$ & 488 & 361 \\
\hline $\begin{array}{l}\text { Individuos que utilizan o han utilizado alguna } \\
\text { vez los servicios sociales }\end{array}$ & 1.320 & 1.406 \\
\hline
\end{tabular}

Fuente: Elaboración propia a partir de las encuestas Foessa 2007-2009 mediante la agrupación de los casos que han utilizado los servicios sociales públicos, Cáritas o a los de otras entidades en busca de ayuda. 
Aunque los análisis efectuados se limitan a la primera parte del período de la crisis económica (2007-2009), la realización de otra encuesta nacional en el año 2013 por parte de la Fundación FOESSA permitiría replicar estos mismos análisis para la sub muestra de usuarios de servicios sociales, y de esta manera comprobar los cambios en las condiciones de vida entre los usuarios de servicios sociales y de la población española.

Siguiendo el esquema de las encuestas de condiciones de vida y de exclusión social, el hogar y el individuo han sido las unidades empleadas en el análisis de las necesidades de esta población (Atkinson et al, 2002; Laparra, y Pérez, 2010 (Coord.)). Así pues, los principales riesgos se han abordado mediante la comparación entre la población usuaria y no usuaria de los servicios sociales.

Al mismo tiempo, los análisis descriptivos realizados en dos momentos del tiempo posibilitan el estudio de las necesidades y las demandas en materia de política social. En último lugar, el proceso de operacionalización llevado a cabo mediante la creación de las dimensiones e indicadores incluye las relaciones sociales, el empleo, los ingresos, la salud y la cobertura de los servicios sociales (Atkinson et al, 2002; Paugam, 1993).

\subsection{Dimensiones, sub-dimensiones e indicadores empleados}

Mediante el proceso de operacionalización (dimensiones, sub-dimensiones e indicadores) se ha analizado las necesidades de los usuarios de servicios sociales y en los cambios experimentados por la población española. A modo de resumen, en este estudio se han considerado las siguientes dimensiones: el perfil de los beneficiarios de servicios sociales, el carácter hereditario de la pobreza, los problemas residenciales, la salud, las relaciones de solidaridad, la protección familiar, la pobreza relativa, las tendencias en el empleo y el desempleo, la cobertura del sistema de protección social, así como de la opinión y los cambios experimentados en el período de estudio.

a) Perfil de beneficiarios de los servicios sociales. Se ha desagregado en tres sub-dimensiones. La primera sub dimensión ha recogido el origen de procedencia, el sexo o la edad, siendo de utilidad en la detección de los cambios en los patrones de la pobreza. La segunda profundiza en la gravedad de los problemas existentes en el hogar, tales como la exclusión moderada, exclusión severa, la integración precaria y la integración social (Laparra, 2010). La tercera sub-dimensión distingue la duración en el tiempo de los itinerarios, 
diferenciando entre antiguos, permanentes y nuevos usuarios de los servicios sociales (García y Karzepov, 2004; Carbonero, 2014).

b) Reproducción y la transmisión intergeneracional de la pobreza. El indicador recoge el nivel de estudios básicos alcanzado por padres e hijos, identificando el carácter hereditario de la pobreza (Esping Andersen, 2002, 2005; Paugam, 2007).

c) Vivienda y alojamiento. Las clasificaciones residenciales se han adaptado al estudio de las necesidades de las personas y de los hogares con mayores riesgosde exclusión social, dando lugar a dos sub dimensiones (Kemeny, 1993; Cortés, 2003).

La primera sub-dimensión incluye los problemas de acceso, estabilidad, adecuación y habitabilidad, surgidos en el ámbito del alojamiento. Por un lado, los problemas de acceso y de estabilidad recogen situaciones ligadas a hogares con problemas de amenaza y expulsión, gastos excesivos, problemas económicos e impagos, así como de aquellos hogares que se han visto obligados a recurrir al patrimonio para hacer frente a los costes de la vivienda. Por otro lado, los problemas de adecuación se han relacionado con la proporción de personas en situación de hacinamiento sociológico, mientras que los problemas de habitabilidad recogen al conjunto de personas residentes en viviendas que carecen de equipamientos básicos o en edificios que no reúnen los requisitos de habitabilidad

La segunda sub-dimensión engloba las dinámicas del hogar y los cambios en la convivencia. Los indicadores empleados han detectado principalmente situaciones de gravedad tales como el cambio de vivienda, el retorno a la casa de los padres, la necesidad de compartir piso o el realquiler de alguna habitación a personas con las que no se comparte parentesco. Por último, el entorno se ha incluido como parte de los problemas residenciales de los usuarios de servicios sociales, considerando los barrios degradados y aquellos que disponen de equipamientos básicos en mal estado (la iluminación, el acerado y el mobiliario urbano) o que carecen de ellos.

d) Salud. La teoría de necesidades formulada por Doyal y Gough (1995), incluye los problemas de salud como una cuestión fundamental para desarrollar al máximo las capacidades personales. Los indicadores empleados en esta dimensión recogen la tasa de personas que se encuentran afectadas por los problemas de salud y el porcentaje de hogares en los cuales todos sus miembros se encuentran en esta situación. 
e) La solidaridad y el apoyo familiar. Las funciones de solidaridad desempeñadas por la familia han sido un rasgo propio de la protección social y de la prevención de la pobreza más extrema (Castel, 1997). Los indicadores incluidos en esta dimensión contribuyen a estudiarla ausencia de ayuda, la dificultad de las redes familiares para hacer frente a los problemas de exclusión y la protección familiar frente a los riesgos de desempleo. En concreto, la proporción de hogares con todos los activos en desempleo identifica los riesgos del mercado de trabajo respecto de la protección familiar. A su vez, los indicadores relativos a la falta de apoyo socio familiar frente a las necesidades, los problemas de aislamiento social y las relaciones conflictivas agrupan los problemas de funcionamiento de la familia.

f) La distribución de la pobreza. El cálculo del umbral de la pobreza relativa se compone de los ingresos disponibles en el hogar y las unidades de equivalencia referidas al tamaño y la composición. De este modo, el empleo de este indicador permite situar al conjunto de personas que se encuentran debajo del $60 \%$ de la renta disponible.

g) Desprotección en el mercado de trabajo. Los problemas de precariedad y dualización del empleo han centrado los problemas de exclusión social en la sociedad española (Zubero, 2006; Laparra, 2007). Las tendencias recogidas en este apartado hacen referencia a la tasa de paro, la evolución del tiempo de duración y la participación en las actividades formativas por parte de las personas en desempleo (Atkinson et al, 2002).

h) Cobertura del sistema de protección social. Se analiza la protección desempeñada por parte de las políiticas públicas desde el ámbito universal y asistencial. En el ámbito universal las políticas públicas de salud y educación se han estudiado mediante el no acceso al sistema sanitario por parte de las personas con enfermedades graves, la dificultad de acceso a medicamentos, los hogares con abandono del sistema educativo, los hogares con menores no escolarizados, hogares formados por adultos sin estudios entre 16-64 años y la proporción de personas desempleadas que no realizan cursos de formación.

En el ámbito asistencial, la gravedad de las condiciones de vida en los hogares se ha relacionado con la cobertura desde los servicios sociales. El indicador de condiciones de vida elaborado por Laparra (2010) incluye a aquellos hogares que padecen algún tipo de precariedad y de exclusión social. Su aplicación en los hogares y las personas que no han utilizado los servicios sociales puede servir para medir el alcance de las políticas sociales. 
i) Cambios en las necesidades. Las preguntas retrospectivas han recogido el cambio en las percepciones sociales ocasionadas por la crisis entre los usuarios de los servicios sociales sobre las necesidades básicas (alimentación, calzado, transporte público, dietas o medicinas), el ocio (televisión o internet) o la extensión de los problemas de exclusión en familiares y conocidos.

\subsection{Análisis de datos}

La prueba estadística empleada ha sido la chi cuadrado y su significación asociada al $5 \%$ entre los usuarios y los no usuarios de los servicios sociales durante 2007 y 2009. Los análisis se han realizado aplicando un factor de ponderación para cada muestra de estudio. El programa estadístico SPSS versión 20 se ha empleado en la creación de los indicadores correspondientes a las variables y dimensiones descritas en el apartado anterior.

\subsection{Principales limitaciones en el uso de la muestra, los análisis efectuados y} las inferencias hacia los usuarios de los servicios sociales

a) Los indicadores de desempleo de larga duración o la cobertura del sistema educativo hacia los sustentadores de hogares en desempleo han obtenido pocos casos en las encuestas FOESSA. De este modo, la obtención de una muestra mayor mejoraría el análisis sobre las condiciones de vida entre los usuarios de los servicios sociales.

b) La escasa muestra de los tipos de servicios sociales ha dificultado la comparación de las necesidades entre los servicios sociales locales, Cáritas y otras instituciones destinadas a la provisión de ayuda. La obtención de una muestra mayor en cada uno de ellos posibilitaría la detección de casos de mayor o menor gravedad en función del tipo de servicios sociales empleados.

c) El empleo de una sub-muestra extraída de las encuestas FOESSA, no refleja al conjunto de los usuarios de los servicios sociales ni la extrapolación al conjunto de esta población. Este hecho evidencia la falta de fuentes de información actualizadas y la ausencia de una encuesta de exclusión social destinada exclusivamente a los usuarios de servicios sociales. 


\section{Evolución y riesgos dominantes en los hogares beneficiarios de los servicios sociales}

\section{I. Cambios en los patrones demográficos y las amenazas del desempleo}

La literatura actual ha identificado los riesgos propios de la pobreza en los hogares monoparentales, así como la precariedad y el desempleo entre los jóvenes que se encuentran sin empleo $o$ en condiciones de fuerte precariedad laboral (Valls, 2010; Brunet, Valls, Belzunegui, 2013; Taylor-Gooby, 2004). Atendiendo al perfil demográfico, estos riesgos se intensifican entre las mujeres y las personas de personas de mediana edad entre los usuarios de los servicios sociales, así como el incremento de 30 a 44 años en 2009 respecto al año 2007 (Tabla 2).

A través de la Encuesta de población activa (EPA) se constata el aumento del paro y su impacto en los hogares que cuentan con todos sus miembros en desempleo (Atkinson et al, 2002). Este hecho amenaza el equilibrio entre la familia y el mercado de trabajo, constatándose el incremento continuado por los sustentadores principales y de los hogares que cuentan con todos sus miembros activos en desempleo ${ }^{3}$ (Tezanos, 1998). Finalmente, las encuestas FOESSA recogen estas tendencias, reflejadas en el crecimiento continuado de los sustentadores del hogar en situación de desempleo tanto en la población usuaria, como entre la no usuaria de los servicios sociales (Tabla 2).

4.2. Alcance de la protección social en el hogar: límites en los ámbitos educativo, sanitario y en los servicios sociales

Los límites más destacados en las políticas universales aparecen en la comparación entre los beneficiarios y no beneficiarios de los servicios sociales, detectándose las tasas más elevadas de abandono educativo entre los hogares formados por adultos sin estudios y de aquellos que no pueden comprar las medicinas necesarias. Por el contrario, se ha detectado una cobertura similar entre los usuarios y no usuarios de los servicios sociales, no obteniendo diferencias significativas entre los hogares que cuentan con menores no escolarizados y aquellos hogares en los que se encuentran personas dependientes sin ningún tipo de apoyo (Tabla 3).

${ }^{3}$ Los sustentadores principales del hogar han aumentado en más del doble entre el primer trimestre de 2005 y el de 2010 , creciendo del $3.7 \%$ al $10.1 \%$, mientras que el aumento de los hogares con todos sus miembros activos en desempleo ha mostrado una tendencia similar, pasando del 4.2 al $10 \%$. 
Sin embargo, los indicadores muestran las mayores dificultades de los servicios sociales en la cobertura de aquellos hogares afectados por algún problema de pobreza y de exclusión social. A partir de la clasificación realizada por Laparra (2010), más de la mitad de los hogares que nunca han acudido a los servicios sociales, tienen problemas de integración social o se encuentran afectados por algún problema de exclusión social. Entre los hogares no usuarios de los servicios sociales se observa un incremento de aquellos en situación de integración precaria y que padecen problemas de exclusión social en 2009 con respecto a 2007 (Tabla 2).

\subsection{Principales necesidades residenciales en los hogares beneficiarios}

Los problemas de la vivienda y del alojamiento crecen notablemente en el conjunto de los hogares españoles, incrementándose la carencia material. En este contexto de crisis, los informes de Eurostat (2103) y las encuestas de condiciones de vida realizadas entre 2005-2010 por el INE (ECV-INE) reflejan las mayores dificultades de los hogares españoles comparativamente en Europa, así como el crecimiento de los problemas de alojamiento en el contexto español. Las problemáticas residenciales recogen el aumento constante de los hogares con dificultades para afrontar gastos imprevistos, el retraso en el pago de la vivienda, las personas residentes en alojamientos con problemas de habitabilidad y las dificultades de los hogares para abordar los gastos de la vivienda de acuerdo a los ingresos disponibles ${ }^{4}$.

En cuanto a los hogares usuarios de los servicios sociales se han visto incrementados los riesgos de carácter residencial entre 2007-2009, materializados en el aumento de los problemas económicos y de impagos, la carencia de viviendas sin equipamientos básicos y los cambios en la composición del hogar. Además, los problemas económicos (acceso y estabilidad) y los cambios en la composición de los hogares se triplican entre aquellos hogares que alguna vez han sido beneficiarios de los servicios sociales. A su vez, estos hogares se duplican en comparación a la población que nunca ha usado los servicios sociales en los indicadores relacionados con el área degradada y la ausencia de equipamientos básicos en la vivienda (Tabla 3).

\footnotetext{
${ }^{4}$ Las dificultades para mantener una vivienda adecuada y el retraso en el pago de gastos relacionados con la vivienda se han reflejado en el conjunto de los hogares españoles. El primero de estos indicadores ha pasado de un $6.2 \%$ a un $7.5 \%$, mientras que el segundo de ellos se ha incrementado de un $5.5 \%$ a un $8.7 \%$. Además, como dato ilustrador de este problema se encuentra la imposibilidad de los hogares para afrontar gastos imprevistos, incrementándose en 10 puntos desde el año 2008 (29,9\%) hasta el año 2010 (38,5\%). http://www.ine.es/jaxiBD/tabla.do
} 
Finalmente, los problemas residenciales han evolucionado de forma desigual entre el período 2007-2009. Como aspecto destacable, se encuentra la disminución de aquellos hogares usuarios de servicios sociales situados en entornos no degradados y en espacios públicos sin equipamientos básicos o en muy mal estado. Mientras que en el año 2007 se alcanzan un $27.7 \%$ y un $54.6 \%$ en los anteriores problemas, en el año 2009 , esta proporción obtiene un $9 \%$ y un $34.3 \%$ (Tabla 3).

\section{Evolución y riesgos dominantes en los personas beneficiarias de los servicios sociales}

\section{I. Principales cambios en el perfil demográfico y en la composición de clase social}

Los análisis efectuados han recogido el cambio en el perfil socio demográfico entre los usuarios de los servicios sociales. En primer lugar, el aumento de personas de origen inmigrante vuelve a aparecer en los análisis efectuados y el descenso de la edad. La población nacida fuera de España ha incrementado en más del doble su presencia en los servicios sociales, el incremento de la pobreza infantil entre los menores de 18 años y el aumento de los usuarios entre las franjas de edad de 30 a 44 años ha provocado un cambio en la estructura de la edad entre los usuarios de servicios sociales (Tabla 4).

En segundo lugar, los resultados obtenidos en la EPA entre el 2005 y 2010 apuntan el impacto del desempleo entre los niveles formativos más bajos y las ocupaciones no cualificadas. Las personas analfabetas, aquellas con educación primaria y en las primeras etapas de educación secundaria alcanzan las tasas más elevadas de paro ${ }^{5}$. Al mismo tiempo, el impacto del desempleo ha sido relevante entre las ocupaciones no cualificadas y las cualificadas pertenecientes al sector de la industria manufacturera, los artesanos, la construcción o la minería ${ }^{6}$. Por último,

\footnotetext{
${ }^{5}$ Atendiendo al nivel de formación, el desempleo en la EPA ha alcanzado las siguientes tasas durante el primer trimestre de 2005 y de 2010 , respectivamente entre las personas analfabetas (27.1\%-47.3\%), la educación primaria (11.3\%-29.4\%), la primera etapa de la educación secundaria (2\%-25.6\%), la segunda etapa de educación secundaria (10.3\%-18.9\%) y la educación superior (7.5\%-11.4\%).

${ }^{6}$ Las tasas de desempleo se han distribuido desigualmente entre el primer trimestre de 2005 y de 2010 , en función de las ocupaciones, situándose el incremento más importante entre los trabajadores cualificados en la industria y la construcción $(5,8 \%-20.1 \%)$ y los trabajadores no cualificados $(11,7 \%-21.8 \%)$.
} 
las series de la EPA revelan la expansión del desempleo de larga duración así como la elevada proporción de personas que no han realizado ningún curso de formación durante el último año?.

Un último aspecto recogido en las encuestas FOESSA ha sido el cambio producido en el mercado de trabajo entre la población usuaria de los servicios sociales. Los riesgos específicos entre la población incorporada entre 2007 y 2009 están principalmente unidos al crecimiento del bajo nivel de estudios, el aumento del desempleo de corta y de larga duración, así comola falta de cobertura del sistema educativo respecto del desempleo.

\subsection{Transmisión intergeneracional, reproducción de la pobreza y las relaciones de solidaridad}

Las tendencias de reproducción y transmisión intergeneracional de los niveles educativos más bajos entre padres e hijos muestran la relevancia de la pobreza en las sociedades pre modernas, alcanzando los resultados más elevados entre las personas que han usado alguna vez los servicios sociales (44.6\%) (Del Pino, y Bericat, 1998) (Tabla 5).

La comparación entre usuarios y no usuarios ha señalado la no existencia de diferencias significativas en cuanto a las personas que carecen de apoyo en situación de necesidad. Si bien, los resultados obtenidos en cuanto a los problemas de aislamiento en el hogar y la familia han sido estadísticamente significativos, las diferencias observadas no han sido tan elevadas como en otros de los indicadores elaborados (Tabla 5).

Finalmente, la importancia de las relaciones familiares y de solidaridad en el hogar se ha reflejado en la comparación entre los usuarios y los no usuarios de los servicios sociales. La suma de los problemas de aislamiento y de conflictos socio familiares es algo mayor entre los usuarios de los servicios sociales. Sin embargo, entre el período 2007 y 2009 , estas tasas han descendido en ambos grupos de población, alcanzado resultados similares en el último período (Tabla 5).

${ }^{7}$ Los desempleados con menos de 6 meses de búsqueda de empleo y más de dos años se distribuyen desigualmente en los trimestres considerados, de tal modo que durante el primer trimestre de 2005 los resultados alcanzan el $46.6 \%$ y el $21 \%$ respectivamente, mientras que en el primer trimestre de 2010 , disminuyen los desempleados de corta duración y aumentan de larga duración, alcanzando respectivamente el $35.5 \%$ y el $38.7 \%$. La falta de cobertura del sistema educativo respecto de la población desempleada, superan el $70 \%$ en los dos períodos de tiempo considerados. 


\subsection{Otras necesidades detectadas: pobreza, salud y alojamiento}

A partir de las ECV- INE, las tasas de pobreza en España afectan a algo menos de la cuarta parte de la población española, alcanzando el $20.1 \%$ y el $21.4 \%$ en la ECV-INE 2005 y la ECV-INE 2009, respectivamente. Sin embargo, las riesgos de situarse debajo del umbral medio de la pobreza afectan en mayor medida a las personas que han utilizado los servicios sociales alguna vez $(30.8 \%)$, que a aquellos que nunca los han usado (14\%) (Tabla 5).

Los problemas de salud se han hecho más evidentes entre las personas que han utilizado los servicios sociales, triplicando a la población que nunca los ha utilizado, alcanzando el $16.5 \%$ en los usuarios y el $4.9 \%$ en los no usuarios en el año 2007 , y el $17.4 \%$ y el $6.8 \%$ en el año 2009 . Finalmente, las tasas de hacinamiento moderado y grave, han decrecido entre 2005-2010 $0^{8}$. Sin embargo, el indicador de hacinamiento calculado a partir de las encuestas FOESSA 9 , muestra los mayores riesgos ente la población usuaria de los servicios sociales, duplicándose estas diferencias en el año 2009 (Tabla 5).

Los cambios en las condiciones de vida y de exclusión social se han reflejado en la opinión pública y en el descenso en el nivel de vida experimentado por la población. El agravamiento en las condiciones de vida de las personas en este período de tiempo se ha materializado en el conjunto de personas que reconocen que la crisis les ha afectado mucho, o que conoce situaciones en las que la crisis les ha afectado gravemente, alcanzando a más de la mitad de la población no usuaria $(60 \%$ y a la mayor parte de la población usuaria de los servicios sociales (90\%) (Tabla 5).

\section{Conclusiones}

Siguiendo el marco de estudio planteado, se aprecia el desequilibrio de las diferentes esferas de bienestar, ocasionado por los cambios en las condiciones de vida en España. Los análisis efectuados apuntan al crecimiento de los riesgos posindustriales,

${ }^{8}$ Los niveles de hacinamiento incluidos en los análisis de las encuestas de condiciones de vida del INEque se han considerado son la suma de la densidad crítica (menos de 0,5 habitaciones por personas) y la densidad moderada (entre 0,5 y 1 habitación persona), alcanzando al $22.4 \%$ y al $14.4 \%$

9 El indicador calculado en la medición de hacinamiento hace referencia a la proporción de personas que disponen de menos de 15 metros cuadrados por persona.

Revista de Fomento Social 70 (2015) 
afectando a la producción de bienestar en los regímenes de la Europa del sur y al desequilibrio entre la familia, la protección social y el mercado de trabajo. En este marco de estudio, las tendencias de las encuestas FOESSA coinciden con los cambios destacados en las series de la EPA y las encuestas de condiciones de vida-

Los problemas residenciales y de desempleo aparecen como las grandes amenazas a la producción de bienestar. Concretamente, su impacto acentúa el desequilibrio existente entre la familia y el mercado de trabajo. Específicamente, el impacto en los indicadores de solidaridad familiar condiciona el papel de la familia como principal agente de provisión de bienestar en España. A su vez, los sistemas de protección universal nos han ofrecido una doble visión respecto de la protección social desempeñada por el sistema sanitario y el sistema educativo. De este modo, la mezcla entre estabilidad y protección en este período de tiempo, se combinan con el incremento de riesgos en indicadores concretos, desafiando el papel redistributivo del Estado de Bienestar y a la cobertura de los riesgos sociales.

Las conclusiones extraídas de los análisis de la sub muestran de usuarios apuntan hacia dos aspectos necesarios de estudio en el ámbito de los servicios sociales y de la protección social: el aumento de los grupos de población más desfavorecidos y el cambio de patrón de las necesidades. El primero de los aspectos recoge un crecimiento de la población usuaria con las ocupaciones y los niveles educativos más bajos. Si bien, este aspecto debe de estudiarse con mayor profundidad, puesto que es necesario saber si este cambio es reflejo de un problema en el tiempo, o por el contrario recoge un problema más duradero, reflejando la expulsión que ha experimentado la población con menor nivel educativo del mercado de trabajo durante el el transcurso del período de crisis económica.

El segundo de los aspectos muestra las diferencias en la composición demográfica y en las relaciones de solidaridad, las cuales revelan un posible cambio de patrón en las necesidades entre la población que acude a los servicios sociales. Por un lado, los cambios más relevantes reflejan el incremento de la población joven, los menores de edad y las personas de origen inmigrante. Su mayor presencia refleja el impacto de la crisis económica sobre la composición de los nuevos usuarios de servicios sociales durante el primer período de crisis económica. Por otro lado, las condiciones de solidaridad y apoyo social han evolucionado en el transcurso de este período de tiempo, mostrando semejanzas entre la población no usuaria de los servicios sociales y el conjunto de la población española, así como de la entrada de población más normalizada a los servicios sociales. 


\section{Bibliografía}

Abrahamson, A. (1997) "Exclusión social en Europa: ìvino viejo en odres nuevos?", en Moreno L. (Coord). Unión Europea y Estado del bienestar. Madrid. CSIC. Colección Politeya.

Atkinson, A. Cantillon, B. Marler, E. y Nolan, B. (2002) Social Indicators. The EU and Social Inclusion. Oxford. Oxford University Press.

Bourdieu, P. y PASSERON, J. C. (1969) Los estudiantes y la cultura. Barcelona. Labor. Carbonero, D. (2014) "Transformaciones en las necesidades de los usuarios de servicios sociales entre 2005-2009". Documentación Social número, $n^{\circ}$ 170, pp 192-216.

CASADO, D. (1996) Introducción a los Servicios sociales. Madrid. Popular.

CASTEL, R. (1997) La metamorfosis de la cuestión social: una crónica del asalariado. Barcelona. Paidós.

Cortés Alcalá, L. Fernández López, C. y Plaza GutiérRez, P. (2003) “Vivienda y Exclusión Residencial", en Laparra, M., Gaviria, M. y Agullar, M. (Coords). Investigaciones de base para la elaboración del Plan de Lucha contra la Exclusión Social en la Comunidad de Madrid. Madrid. Universidad Pública de Navarra y Comunidad de Madrid.

Del PINO, J. y Bericat, E. (1998) Valores sociales en la cultura andaluza. Encuesta Mundial de valores. Andalucía 1996. Madrid. CIS. Monografías número 156.

DoyAl, L. y Gough, I. (1995) Teoría de las necesidades humanas. Madrid. IcariaFuhem. Madrid.

Durkheim, E. (1987) La división del Trabajo Social. Madrid. Akal. Colección Universitaria.

Esping Andersen, G. (2002) Fundamentos Sociales de las economías post industriales. Madrid. Ariel.

- (2005) "Invertir en los niños y combatir la herencia social, en López GANDía, J. y Ochando Claramunt, C. (Coords). El Estado del bienestar: modelos y líneas de reforma. Valencia. Colección Ciencias del Trabajo. 
EUROSTAT (2013). European social statistics edition. Bruselas. Pocketbooks.http:// www.siis.net/es/documentaciondetalle. $p h p ? i d=2747 \&$ tema $=0 \& m e s=12 \& a n i o=$ 2013\&palabra.

García, S. y Karzepov, Y. (2004) "Perché alcune persone hanno più probabilità di essere in assistenza di altri", en SARRACERNO (Coord.): Le dinamiche assistenziali in Europa. Bolonia. II Mulino.

KEMENY, J. (1992) Housing and Social Theory. Londres. Routledge.

Laparra, M. (2002) "Una perspectiva de conjunto sobre el espacio social de la exclusión social", en Moreno, L (Coord). Pobreza y exclusión social: La malla de seguridad en España. Madrid. CSIC.Colección Politeya.

- (2007) La construcción del empleo precario: dimensiones, causas y tendencias de la precariedad laboral. Madrid. Fundación Foessa.

- (2010) "Alteraciones en el espacio social de la exclusión en un contexto de crisis económica", en Laparra, M. y Pérez, B. (Coords) El primer impacto de la crisis en la cohesión social en España. Madrid. Fundación Foessa. Cáritas.

Laparra, M y Pérez, B. (2010) (coordinadores) El primer impacto de la crisis en la cohesión social en España. Madrid. Fundación Foessa. Madrid. Cáritas.

MeULman y HeISER (2005) SPSS categories user's manual v140. Chicago. SPSS.

Marshall, T. H. y Botтomore, T. (1950) Citizenship and Social Class and Other Essays, Cambridge. Cambridge UniversityPress.

PARDO, A y RUIZ, M. (2002) SPSS1 1: Guía para el análisis de datos. Madrid. Mc-Graw-Hill.

Paugam, S. (1993) La disqualification sociale. Essai sur la nouvelle pauvreté. Paris. Presses Universitaires de France.

- (2007) Las formas elementales de la pobreza. Madrid. Alianza.

Rodríguez Cabrero, G. (2005) "El Estado de bienestar en España: procesos de reestructuración, impactos sociales y tendencias de desarrollo en la era de la globalización. Corintios XIII: Revista de teología y pastoral de la Caridad, $\mathrm{n}^{\circ}$ 112-113, pp. 69-124. 
RodríGuez, M. J. y NaVArRo, C. J. (2008) "El esfuerzo público de des familiarización. Estudio Comparado en la Unión Europea". Papers, n 90: pp. 59-81.

SarRaCerno, C. (2004) (Coord.): Le dinamiche assistenziali in Europa. Bolonia. IIMulino.

TAYLOR-GOOBY, P. (2004) “Nuevos riesgos sociales en la sociedad post-industrial: algunas evidencias del Eurobarómetro acerca de las respuestas a las políticas activas del mercado de trabajo". Revista Internacional de Seguridad Social. Vol. $57, N^{\circ} .3$, pp. $51-74$.

Vaus, F. (2010) "La pobreza de las juventudes: análisis de las formas elementales de pobreza juvenil en España". Empiria n ${ }^{\circ} 21$, pp. 97-120.

ZUBERO, I. (2006) "Las nuevas relaciones entre empleo e inclusión: flexibilización del trabajo y precarización vital". Documentación Social, n 143, pp. 11-30. 


\begin{tabular}{|c|c|c|c|c|c|c|c|c|c|c|}
\hline \multicolumn{11}{|c|}{$\begin{array}{l}\text { ANEXO I } \\
\text { TABLA 2. Diferencias demográficas, labo } \\
\text { rios de servicios sociales (2007-2009) }\end{array}$} \\
\hline & & & & & \multirow{2}{*}{$\begin{array}{c}\text { Pvalor } \\
+\end{array}$} & & & & & \multirow{2}{*}{$\begin{array}{c}P \text { Palor } \\
++\end{array}$} \\
\hline & $N$ & $\%$ & $N$ & $\%$ & & $N$ & $\%$ & $N$ & $\%$ & \\
\hline $\begin{array}{l}\text { Sexo } \\
\text {-Hombres } \\
\text {-Mujeres } \\
\end{array}$ & $\begin{array}{l}147 \\
341 \\
\end{array}$ & $\begin{array}{l}30.2 \% \\
69.8 \% \\
\end{array}$ & $\begin{array}{l}1.215 \\
1.713 \\
\end{array}$ & $\begin{array}{l}41.5 \% \\
58.5 \% \\
\end{array}$ & 0.000 & $\begin{array}{l}181 \\
273 \\
\end{array}$ & $\begin{array}{l}30.4 \% \\
69.6 \% \\
\end{array}$ & \begin{tabular}{|l}
1.254 \\
1.893 \\
\end{tabular} & $\begin{array}{l}41.2 \% \\
58.8 \% \\
\end{array}$ & 0.000 \\
\hline $\begin{array}{l}\text { Edad } \\
-18-29 \\
-30-44 \\
-45-64 \\
-65 \text { y más }\end{array}$ & $\begin{array}{c}56 \\
102 \\
205 \\
126 \\
\end{array}$ & \begin{tabular}{|l|}
$11.5 \%$ \\
$20.8 \%$ \\
$41.4 \%$ \\
$25.8 \%$ \\
\end{tabular} & $\begin{array}{l}446 \\
874 \\
923 \\
685 \\
\end{array}$ & $\begin{array}{l}15.2 \% \\
29.8 \% \\
31.5 \% \\
23.4 \% \\
\end{array}$ & 0.000 & $\begin{array}{c}46 \\
140 \\
142 \\
126 \\
\end{array}$ & \begin{tabular}{|l|}
$10.4 \%$ \\
$30.8 \%$ \\
$31.2 \%$ \\
$27.4 \%$ \\
\end{tabular} & $\begin{array}{l}347 \\
846 \\
999 \\
954 \\
\end{array}$ & $\begin{array}{l}11.1 \% \\
26.9 \% \\
31.7 \% \\
30.3 \% \\
\end{array}$ & 0.406 \\
\hline $\begin{array}{l}\text { País de nacimiento } \\
\text {-Española } \\
\text {-Extracomunitario }\end{array}$ & $\begin{array}{c}436 \\
52 \\
\end{array}$ & \begin{tabular}{|l}
$89.4 \%$ \\
$10.6 \%$ \\
\end{tabular} & $\begin{array}{c}250 \\
2.675 \\
\end{array}$ & \begin{tabular}{|c|}
$11.5 \%$ \\
$8.2 \%$ \\
\end{tabular} & 0.000 & $\begin{array}{l}341 \\
113 \\
\end{array}$ & \begin{tabular}{|l|}
$75.1 \%$ \\
$24.6 \%$ \\
\end{tabular} & \begin{tabular}{|c|}
2.278 \\
370 \\
\end{tabular} & \begin{tabular}{|l|}
$88.2 \%$ \\
$11.8 \%$ \\
\end{tabular} & 0.000 \\
\hline $\begin{array}{l}\text { Tamaño del hogar } \\
\text {-Persona sola } \\
-2 \text { miembros } \\
-3-4 \text { miembros } \\
-5 \text { miembros o más } \\
\end{array}$ & $\begin{array}{c}94 \\
122 \\
241 \\
31 \\
\end{array}$ & \begin{tabular}{|c}
$19.3 \%$ \\
$25 \%$ \\
$49.9 \%$ \\
$5.8 \%$ \\
\end{tabular} & $\begin{array}{c}530 \\
931 \\
1.270 \\
198 \\
\end{array}$ & $\begin{array}{c}18.1 \% \\
31.8 \% \\
43.3 \% \\
6.8 \% \\
\end{array}$ & 0.018 & $\begin{array}{c}603 \\
1.079 \\
1.242 \\
225 \\
\end{array}$ & \begin{tabular}{|c|}
$15 \%$ \\
$28.2 \%$ \\
$39.1 \%$ \\
$17.7 \%$ \\
\end{tabular} & $\begin{array}{c}68 \\
128 \\
178 \\
80 \\
\end{array}$ & \begin{tabular}{|c|}
$19.1 \%$ \\
$34.3 \%$ \\
$39.4 \%$ \\
$7.2 \%$ \\
\end{tabular} & 0.000 \\
\hline $\begin{array}{l}\text { Estudios del sustentador principal } \\
\text {-Ni lee ni escribe, inferior al graduado escolar y E.S.O. } \\
\text {-Graduado escolar, E.S.O. Bachiller } \\
\text {-BUP, FPI, FPII, Bachiller o superior } \\
\text {-Diplomado, licenciado o superior } \\
\text {-NS/NC/Perdidos }\end{array}$ & $\begin{array}{c}370 \\
333 \\
216 \\
193 \\
95 \\
\end{array}$ & \begin{tabular}{|c|}
$30.7 \%$ \\
$27.6 \%$ \\
$17.9 \%$ \\
$16 \%$ \\
$7.9 \%$ \\
\end{tabular} & $\begin{array}{c}1.851 \\
1.552 \\
1.828 \\
1.261 \\
839 \\
\end{array}$ & \begin{tabular}{|l|}
$25.2 \%$ \\
$21.2 \%$ \\
$24.9 \%$ \\
$17.2 \%$ \\
$11.4 \%$ \\
\end{tabular} & 0.000 & $\begin{array}{c}215 \\
130 \\
74 \\
20 \\
1\end{array}$ & \begin{tabular}{|c|}
$48.9 \%$ \\
$29.5 \%$ \\
$16.8 \%$ \\
$4.5 \%$ \\
$0.2 \%$ \\
\end{tabular} & \begin{tabular}{|c}
1.062 \\
890 \\
674 \\
363 \\
6 \\
\end{tabular} & \begin{tabular}{|c|}
$35.5 \%$ \\
$29.7 \%$ \\
$22.5 \%$ \\
$12.1 \%$ \\
$0.2 \%$ \\
\end{tabular} & 0.000 \\
\hline
\end{tabular}




\begin{tabular}{|c|c|c|c|c|c|c|c|c|c|c|}
\hline $\begin{array}{l}\text { Ocupación del sustentador principal } \\
\text {-Trabajando } \\
\text {-Buscando empleo } \\
\text {-Jubilación, prejubilación u otra pensión } \\
\text {-Otras (Estudiante, labores del hogar, incapacidad y } \\
\text { otras situaciones) }\end{array}$ & $\begin{array}{c}139 \\
42 \\
230 \\
7\end{array}$ & $\begin{array}{l}33.2 \% \\
10.1 \% \\
33.6 \% \\
20.1 \%\end{array}$ & $\begin{array}{l}1.537 \\
116 \\
876 \\
11\end{array}$ & $\begin{array}{c}60.5 \% \\
4.6 \% \\
25.2 \% \\
9.7 \%\end{array}$ & 0.000 & $\begin{array}{l}107 \\
122 \\
115 \\
112\end{array}$ & $\begin{array}{l}24.4 \% \\
26.8 \% \\
25.2 \% \\
23.6 \%\end{array}$ & $\begin{array}{l}1.218 \\
321 \\
891 \\
719\end{array}$ & $\begin{array}{l}28.7 \% \\
10.2 \% \\
28.3 \% \\
22.8 \%\end{array}$ & 0.000 \\
\hline $\begin{array}{l}\text { Desprotección del mercado de trabajo } \\
\text {-Hogares cuyo sustentador principal dejó de tener } \\
\text { empleo desde hace un año o más }\end{array}$ & 6 & $1.2 \%$ & 29 & $1 \%$ & 0.627 & 159 & $11.3 \%$ & 81 & $4.4 \%$ & 0.000 \\
\hline $\begin{array}{l}\text { 1.Integración social } \\
\text { 2.Integración precaria } \\
\text { 3.Exclusión moderada o compensada } \\
\text { 4.Exclusión severa }\end{array}$ & $\begin{array}{c}140 \\
220 \\
83 \\
46\end{array}$ & $\begin{array}{c}28.6 \% \\
45.1 \% \\
17 \% \\
9.3 \%\end{array}$ & $\begin{array}{c}1.530 \\
971 \\
303 \\
125\end{array}$ & $\begin{array}{c}52.3 \% \\
33.2 \% \\
10.3 \% \\
4.2 \%\end{array}$ & 0.000 & $\begin{array}{c}68 \\
227 \\
90 \\
70\end{array}$ & $\begin{array}{l}14.9 \% \\
49.9 \% \\
19.8 \% \\
15.4 \%\end{array}$ & $\begin{array}{c}1.330 \\
1.416 \\
310 \\
93\end{array}$ & $\begin{array}{c}42.2 \% \\
45 \% \\
9.8 \% \\
3 \%\end{array}$ & 0.000 \\
\hline
\end{tabular}

Fuente: Elaboración propia a partir de los módulos transversales de las encuestas Foessa 2007 y 2009

p valort: valor de la comparativa en la distribución entre hogares usuarios y no usuarios en 2007. p valor ++: valor de la comparativa en la distribución entre hogares usuarios y no usuarios en 2009. 


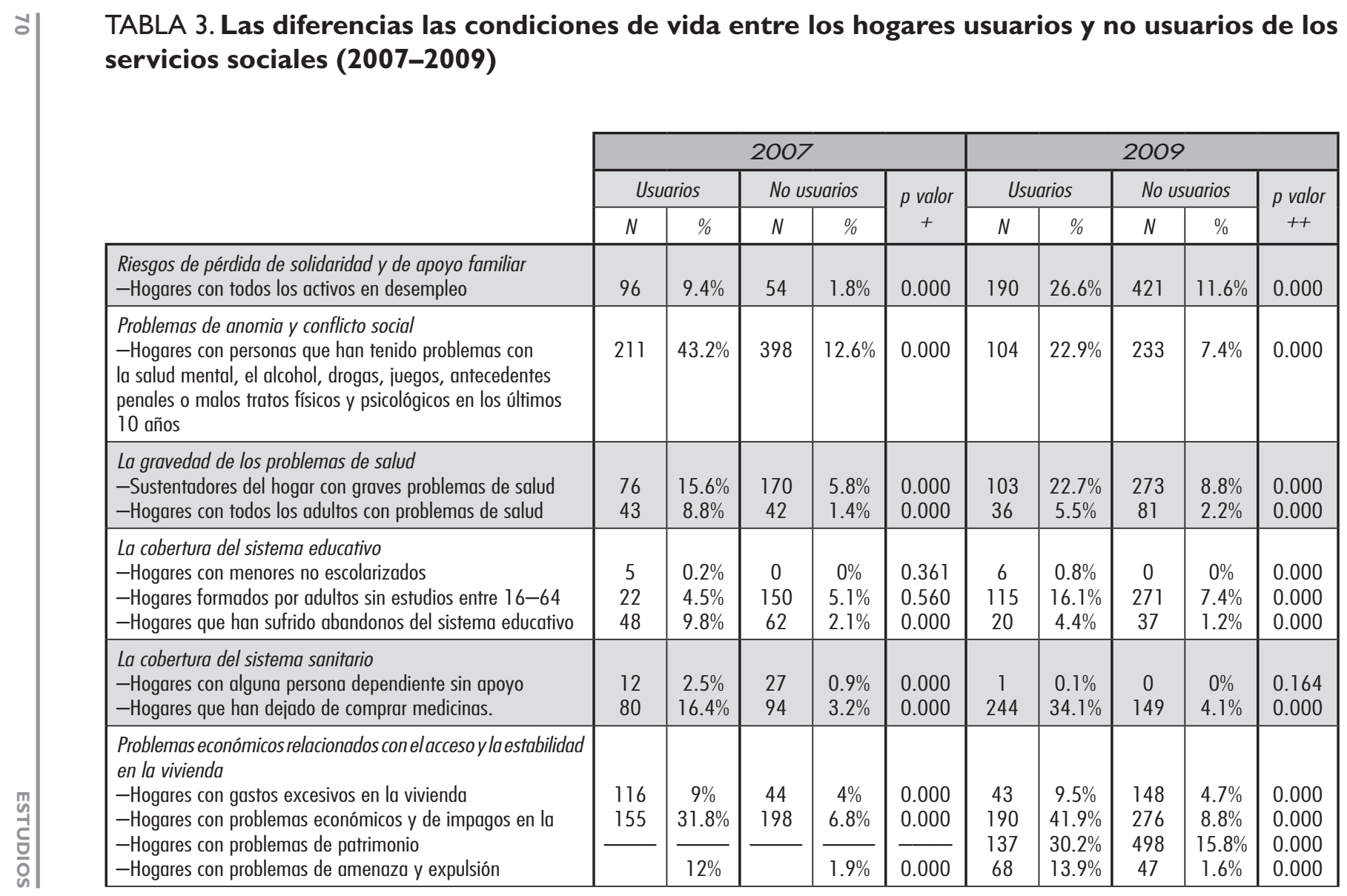




\begin{tabular}{|c|c|c|c|c|c|c|c|c|c|c|}
\hline $\begin{array}{l}\text { Problemas de inhabitabilidad de la vivienda. } \\
\text {-Deficiencias e insalubridad. } \\
\text {-Ausencia de bienes y de equipamientos básicos }\end{array}$ & $\begin{array}{l}74 \\
12\end{array}$ & $\begin{array}{c}15.2 \% \\
2.5 \%\end{array}$ & $\begin{array}{l}290 \\
117\end{array}$ & $\begin{array}{c}9.9 \% \\
4 \%\end{array}$ & $\begin{array}{l}0.000 \\
0.141\end{array}$ & $\begin{array}{l}36 \\
32\end{array}$ & $\begin{array}{l}8.1 \% \\
7.2 \%\end{array}$ & $\begin{array}{l}232 \\
221\end{array}$ & $\begin{array}{l}7.4 \% \\
7.1 \%\end{array}$ & $\begin{array}{l}0.639 \\
0.000\end{array}$ \\
\hline $\begin{array}{l}\text { Cambios en la composición del hogar } \\
\text {-Problemas residenciales asociados al cambio de vivienda, } \\
\text { volver a vivir a la casa de mis padres, compartir piso con } \\
\text { personas sin parentesco, alquilar alguna habitación a otros }\end{array}$ & 37 & $7.6 \%$ & 94 & $3.2 \%$ & 0.000 & 66 & $14.5 \%$ & 146 & $4.6 \%$ & 0.000 \\
\hline $\begin{array}{l}\text { Área y entorno de la vivienda } \\
\text { - Hogares y viviendas en entornos degradados o muy degradados } \\
\text { - Hogares y viviendas situadas en espacios públicos sin } \\
\text { equipamientos o en mal estado }\end{array}$ & $\begin{array}{l}135 \\
256\end{array}$ & $\begin{array}{l}27.7 \% \\
54.6 \%\end{array}$ & $\begin{array}{l}336 \\
233\end{array}$ & $\begin{array}{l}11.5 \% \\
27.1 \%\end{array}$ & $\begin{array}{l}0.000 \\
0.000\end{array}$ & $\begin{array}{c}41 \\
154\end{array}$ & $\begin{array}{c}9 \% \\
34.3 \%\end{array}$ & $\begin{array}{l}191 \\
614\end{array}$ & $\begin{array}{l}6.1 \% \\
20 \%\end{array}$ & $\begin{array}{l}0.024 \\
0.000\end{array}$ \\
\hline
\end{tabular}

Fuente: Elaboración propia a partir de los módulos transversales de las encuestas Foessa 2007 y 2009 p valor+: valor de la comparativa en la distribución entre hogares usuarios y no usuarios en 2007. p valor ++: valor de la comparativa en la
distribución entre hogares usuarios y no usuarios en 2009 . 


\begin{tabular}{|c|c|c|c|c|c|c|c|c|c|c|}
\hline \multicolumn{11}{|c|}{$\begin{array}{l}\text { ANEXO II } \\
\text { TABLA 4. Diferencias demográficas, laborales, educativas y de salud entre las personas usuarias } \\
\text { y no usuarias de los servicios sociales }\end{array}$} \\
\hline & \multicolumn{5}{|c|}{2007} & \multicolumn{5}{|c|}{2009} \\
\hline & \multicolumn{2}{|c|}{ Usuarios } & \multicolumn{2}{|c|}{ No usuarios } & \multirow{2}{*}{$\begin{array}{c}p \text { valor } \\
+\end{array}$} & \multicolumn{2}{|c|}{ Usuarios } & \multicolumn{2}{|c|}{ No usuarios } & \multirow{2}{*}{$\begin{array}{c}p \text { valor } \\
++\end{array}$} \\
\hline & $N$ & $\%$ & $N$ & $\%$ & & $N$ & $\%$ & N & $\%$ & \\
\hline $\begin{array}{l}\text { Sexo } \\
\text {-Hombres } \\
\text {-Mujeres }\end{array}$ & $\begin{array}{l}613 \\
708\end{array}$ & $\begin{array}{l}46.4 \% \\
53.6 \%\end{array}$ & $\begin{array}{l}3.724 \\
4.046\end{array}$ & $\begin{array}{l}47.9 \% \\
52.1 \%\end{array}$ & 0.305 & $\begin{array}{l}655 \\
751\end{array}$ & $\begin{array}{l}46.6 \% \\
53.4 \%\end{array}$ & \begin{tabular}{|l|}
3.976 \\
4.208
\end{tabular} & $\begin{array}{l}48.6 \% \\
51.4 \%\end{array}$ & 0.166 \\
\hline $\begin{array}{l}\text { Edad } \\
- \text { Menores de } 18 \\
-18-29 \\
-30-44 \\
-45-64 \\
-65 \text { y más }\end{array}$ & $\begin{array}{l}193 \\
273 \\
209 \\
366 \\
279\end{array}$ & $\begin{array}{l}14.6 \% \\
20.7 \% \\
15.8 \% \\
27.7 \% \\
21.1 \% \\
\end{array}$ & $\begin{array}{l}1.234 \\
1.476 \\
1.807 \\
2.003 \\
1.250\end{array}$ & \begin{tabular}{|l}
$15.9 \%$ \\
$19 \%$ \\
$23.3 \%$ \\
$25.8 \%$ \\
$16.1 \%$
\end{tabular} & 0.000 & $\begin{array}{l}336 \\
210 \\
334 \\
289 \\
238\end{array}$ & $\begin{array}{l}23.9 \% \\
14.9 \% \\
23.7 \% \\
20.5 \% \\
16.9 \% \\
\end{array}$ & \begin{tabular}{|l|}
1.204 \\
1.252 \\
1.877 \\
2.124 \\
1.728
\end{tabular} & $\begin{array}{l}14.7 \% \\
15.3 \% \\
22.9 \% \\
25.9 \% \\
21.1 \% \\
\end{array}$ & 0.000 \\
\hline $\begin{array}{l}\text { País de nacimiento } \\
\text {-Española } \\
\text {-Extracomunitario }\end{array}$ & $\begin{array}{c}1.185 \\
132 \\
\end{array}$ & $\begin{array}{l}90 \% \\
10 \% \\
\end{array}$ & $\begin{array}{c}7.081 \\
637 \\
\end{array}$ & $\begin{array}{c}91.7 \% \\
8.3 \%\end{array}$ & 0.033 & $\begin{array}{c}1.047 \\
360 \\
\end{array}$ & $\begin{array}{l}74.4 \% \\
25.6 \% \\
\end{array}$ & $\begin{array}{c}7.287 \\
896\end{array}$ & $\begin{array}{l}89.1 \% \\
10.9 \% \\
\end{array}$ & 0.000 \\
\hline $\begin{array}{l}\text { Estudios } \\
\text {-Ni lee ni escribe, inferior al graduado, escolar, E.S.O. } \\
\text {-Graduado escolar, E.S.O., bachiller } \\
\text {-BUP, FPI, FPll, bachiller o superior } \\
\text {-Diplomado, licenciado o superior } \\
\text {-NS/NC/Perdidos }\end{array}$ & $\begin{array}{c}370 \\
333 \\
216 \\
193 \\
95\end{array}$ & \begin{tabular}{|c|}
$30.7 \%$ \\
$27.6 \%$ \\
$17.9 \%$ \\
$16 \%$ \\
$7.9 \%$
\end{tabular} & $\begin{array}{c}1.851 \\
1.552 \\
1.828 \\
1.261 \\
839\end{array}$ & $\begin{array}{l}25.2 \% \\
21.2 \% \\
24.9 \% \\
17.2 \% \\
11.4 \%\end{array}$ & 0.000 & $\begin{array}{c}472 \\
336 \\
172 \\
42 \\
3\end{array}$ & \begin{tabular}{|c|}
$46 \%$ \\
$32.8 \%$ \\
$16.8 \%$ \\
$4.1 \%$ \\
$0.3 \%$
\end{tabular} & \begin{tabular}{|c|}
2.074 \\
2.057 \\
1.564 \\
754 \\
19
\end{tabular} & \begin{tabular}{|c|}
$32.1 \%$ \\
$31.8 \%$ \\
$24.2 \%$ \\
$11.7 \%$ \\
$0.3 \%$
\end{tabular} & 0.000 \\
\hline
\end{tabular}




\begin{tabular}{|c|c|c|c|c|c|c|c|c|c|c|}
\hline $\begin{array}{l}\text { Situación laboral } \\
\text {-Trabajando } \\
\text {-Buscando empleo } \\
\text {-Jubilación, prejubilación u otra pensión } \\
\text {-Otras (Estudiante, labores del hogar, incapacidad y otras) }\end{array}$ & $\begin{array}{c}333 \\
78 \\
430 \\
18\end{array}$ & $\begin{array}{c}38.8 \% \\
9.1 \% \\
50.1 \% \\
2 \%\end{array}$ & $\begin{array}{l}3.433 \\
256 \\
1.478 \\
60\end{array}$ & $\begin{array}{c}65.7 \% \\
4.9 \% \\
28.3 \% \\
1.1 \%\end{array}$ & 0.000 & $\begin{array}{l}315 \\
312 \\
236 \\
244\end{array}$ & $\begin{array}{c}28.5 \% \\
28.2 \% \\
21.3 \% \\
22 \%\end{array}$ & $\begin{array}{l}3.018 \\
835 \\
1.563 \\
1.708\end{array}$ & $\begin{array}{c}42.4 \% \\
11.7 \% \\
21.9 \% \\
24 \%\end{array}$ & 0.000 \\
\hline $\begin{array}{l}\text { Situación laboral en la última semana } \\
\text {-Personas que trabajan } \\
\text {-Personas paradas que buscan empleo ( } 6 \text { meses) } \\
\text {-Personas paradas que buscan empleo ( } 7-12 \text { meses) } \\
\text {-Personas paradas que buscan empleo (+1 año) } \\
\text {-Personas paradas que no buscan empleo } \\
\text {-Personas inactivas (jubilación o prejubilación) } \\
\text {-Personas inactivas (labores del hogar) } \\
\text {-Otras personas inactivas }\end{array}$ & $\begin{array}{c}333 \\
41 \\
23 \\
8 \\
5 \\
299 \\
76 \\
73\end{array}$ & $\begin{array}{c}38.8 \% \\
4.8 \% \\
2.7 \% \\
0.9 \% \\
0.6 \% \\
34.8 \% \\
8.9 \% \\
8.5 \%\end{array}$ & $\begin{array}{c}315 \\
80 \\
94 \\
87 \\
29 \\
236 \\
138 \\
127\end{array}$ & $\begin{array}{c}65.7 \% \\
1.9 \% \\
0.9 \% \\
0.9 \% \\
0.8 \% \\
19.6 \% \\
7.2 \% \\
3.2 \%\end{array}$ & 0.000 & & $\begin{array}{c}28.5 \% \\
7.2 \% \\
8.5 \% \\
7.9 \% \\
2.6 \% \\
21.3 \% \\
12.5 \% \\
11.5 \%\end{array}$ & $\begin{array}{c}3.021 \\
192 \\
178 \\
306 \\
71 \\
1.567 \\
962 \\
755\end{array}$ & $\begin{array}{c}42.4 \% \\
2.7 \% \\
2.5 \% \\
4.3 \% \\
1 \% \\
22 \% \\
13.5 \% \\
10.6 \%\end{array}$ & 0.000 \\
\hline $\begin{array}{l}\text { Cobertura del sistema educativo } \\
\text {-Proporción de individuos en situación de desempleo que no } \\
\text { ha realizado cursos de formación durante el último año }\end{array}$ & 23 & $70.5 \%$ & 30 & $88.3 \%$ & 0.001 & 296 & $94.9 \%$ & 779 & $93.4 \%$ & 0.141 \\
\hline
\end{tabular}

Fuente: Elaboración propia a partir de los módulos transversales de las encuestas Foessa 2007 y 2009 p valor+: valor de la comparativa en la distribución entre hogares usuarios y no usuarios en 2007. p valor ++: valor de la comparativa en la
distribución entre hogares usuarios y no usuarios en 2009 . 


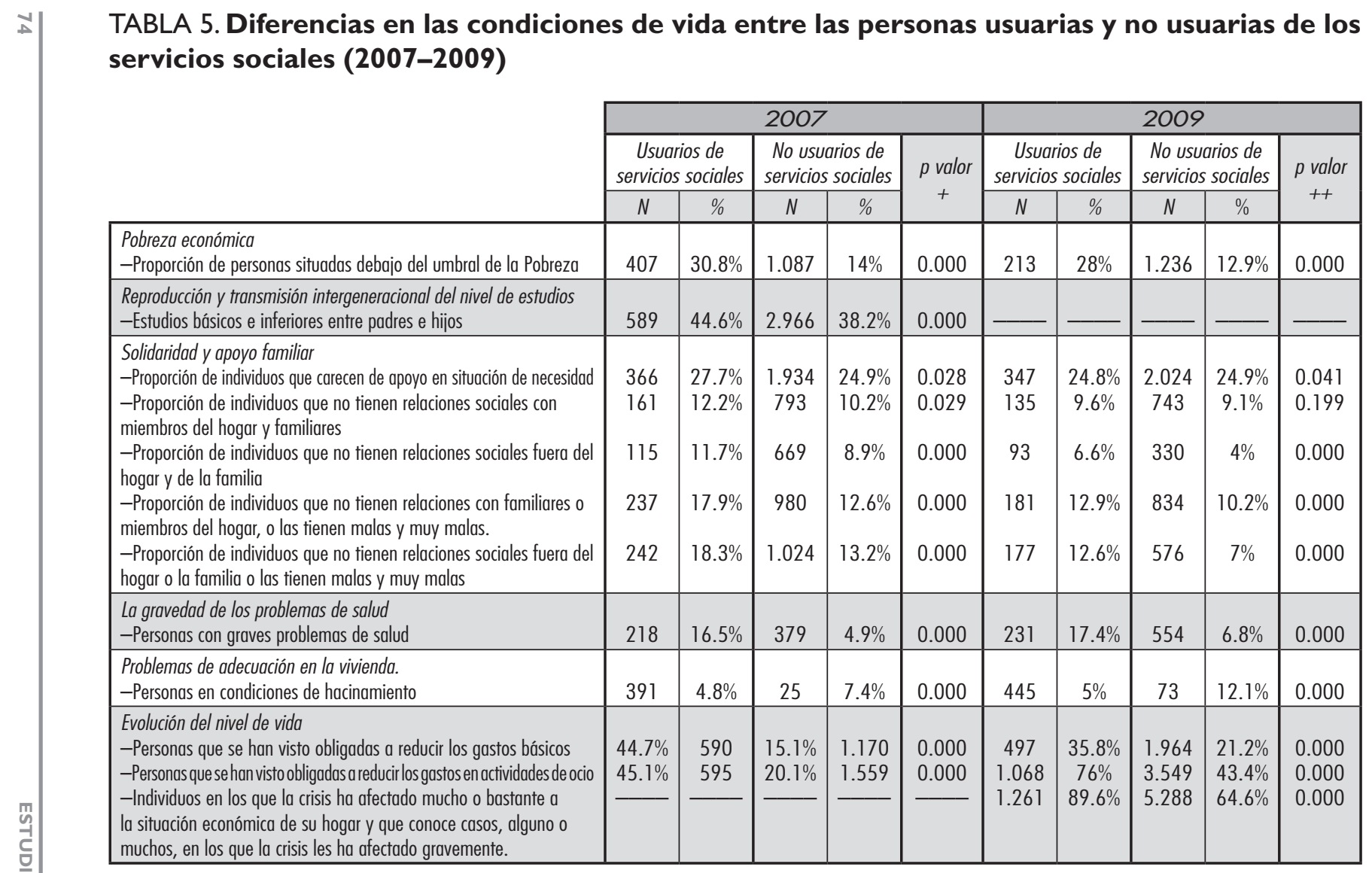

